\title{
Cotranscriptional processes and their influence on genome stability
}

\author{
Xialu Li and James L. Manley ${ }^{1}$ \\ Department of Biological Sciences, Columbia University, New York, New York 10027, USA
}

\begin{abstract}
Numerous studies support the idea that the complex process of gene expression is composed of multiple highly coordinated and integrated steps. While such an extensive coupling ensures the efficiency and accuracy of each step during the gene expression pathway, recent studies have suggested an evolutionarily conserved function for cotranscriptional processes in the maintenance of genome stability. Specifically, such processes prevent a detrimental effect of nascent transcripts on the integrity of the genome. Here we describe studies indicating that nascent transcripts can rehybridize with template DNA, and that this can lead to DNA strand breaks and rearrangements. We present an overview of the diverse mechanisms that different species employ to keep nascent RNA away from DNA during transcription. We also discuss possible mechanisms by which nascent transcripts impact genome stability, as well as the possibility that transcription-induced genomic instability may contribute to disease.
\end{abstract}

Transcripts produced by RNA polymerase (RNAP) II are subject to multiple RNA processing steps in the cell nucleus, including capping, splicing, and polyadenylation, before being transported to the cytoplasm as translatable mRNA. Studies over the last decade have indicated an unanticipated integration of these processes (for reviews, see Hirose and Manley 2000; Maniatis and Reed 2002; Proudfoot et al. 2002). While transcription as well as each step of RNA processing can be carried out independently by distinct machineries in vitro, a growing body of evidence, from both biochemical and genetic assays, has revealed that the pre-mRNA processing events, together with mRNA surveillance and mRNA export from the nucleus, are tightly coordinated with transcription (Fig. 1; for excellent reviews, see Zorio and Bentley 2004; Aguilera 2005a; Reed and Cheng 2005). It has been suggested that such an extensive network of coupling ensures the efficiency and accuracy of each step, and indeed, this undoubtedly is an important aspect of coupling. However, a number of recent studies have revealed

[Keywords: R loop; cotranscriptional RNA processes; genome stability] ${ }^{1}$ Corresponding author.

E-MAIL jlm2@columbia.edu; FAX (212) 865-8246.

Article is online at http://www.genesdev.org/cgi/doi/10.1101/gad.1438306. a second function for this extensive integration: protecting chromosomes from potentially deleterious effects that can arise from interactions between the nascent RNA and template DNA during transcription. In this review, we present an overview of earlier studies as well as recent findings that support the existence of an evolutionarily conserved role for cotranscriptional processes in the maintenance of genome stability. Our focus will be on how different species protect the integrity of their genomes from potentially disastrous effects of nascent transcripts by diverse mechanisms. We also discuss the possible mechanisms by which, if unchecked, nascent transcripts can lead to genome instability. The reader is also referred to related reviews that have appeared recently (e.g., Aguilera 2002, 2005b; Drolet 2006).

\section{The phenomenon of transcriptional $R$ loops}

An R loop is a structure in which an RNA molecule is partially or completely hybridized with one strand of a double-stranded DNA, leaving the other strand unpaired (Fig. 2). Such RNA-DNA hybrids were first observed in vivo at the replication origin of a ColE1-like plasmid to generate a primer for replication initiation (Itoh and Tomizawa 1980). R loops were also observed in early studies of transcription. Over $60 \%$ of RNA transcribed by purified calf thymus RNAP II in vitro was detected in the form of extensive RNA:DNA hybrids following transcription (Kadesch and Chamberlin 1982). In vitro transcription of hypernegatively supercoiled pBR322 plasmid DNA by purified Escherichia coli RNAP also produced RNA:DNA hybrids (Drolet et al. 1994). While this in vitro data has suggested that newly synthesized RNA has the inherent capacity to disturb the duplex DNA template, forming RNA:DNA hybrids behind the elongating RNAP, this appears to occur only occasionally in vivo. Although short stretches of RNA:DNA hybrids have been well documented in the transcription bubble (<20 base pairs) (Gnatt et al. 2001; Kettenberger et al. 2004; Westover et al. 2004), extensive transcriptional R looping has been thought to be relatively rare in vivo except under certain physiological and/or pathological conditions.

Early evidence for in vivo R-loop formation during transcription came from genetic and biochemical studies of the role of topoisomerases in transcription in E. coli 


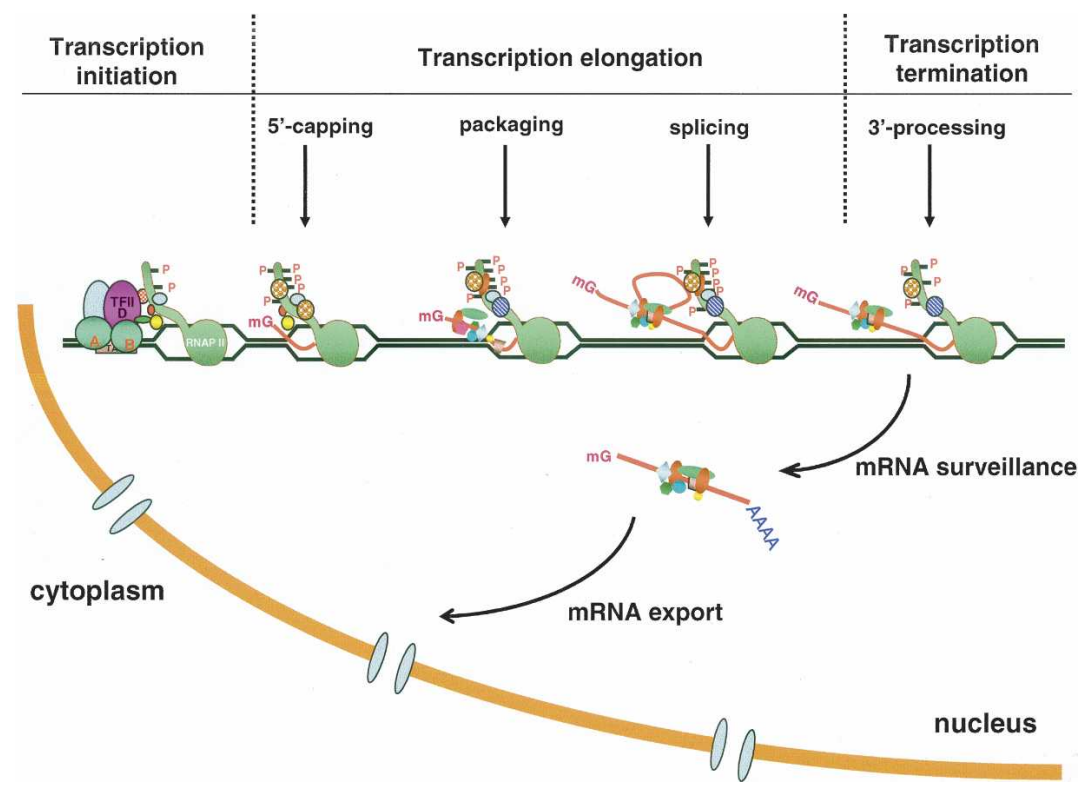

Figure 1. Processes involved in the synthesis of mature mRNA by RNAP II. In eukaryotic cells, the generation of a translatable mRNA is a highly coordinated, multiple-step process that occurs in the nucleus. RNAP II, and specifically the C-terminal domain (CTD) of its largest subunit (indicated by "tail" on the RNAP), orchestrates these processes in a manner that involves changes in CTD phosphorylation status and corresponding changes in associated factors. These proteins in turn effect the subsequent processing reactions and/or help to recruit processing/packaging factors to the nascent transcript. In the process of maturation, the nascent RNA is capped at its $5^{\prime}$ end, introns are removed by splicing, and its $3^{\prime}$ end is cleaved and polyadenylated. After going through the mRNA surveillance system, the matured mRNA is exported to the cytoplasm for translation. Each stage in RNAP II transcription and the steps of cotranscriptional processing are indicated at the top.
(Drolet et al. 1995; Masse et al. 1997; Masse and Drolet 1999|. Based on the formation of RNase H-sensitive hypernegatively supercoiled plasmids and gel retardation assays, persistent $\mathrm{R}$ loops were detected on plasmid DNAs isolated from E. coli topA mutants (Drolet et al. 1994). TopA is the gene encoding DNA topoisomerase I (Topo I), responsible for the relaxation of negative supercoils in E. coli (Drlica 1992). According to the twin-domain model, negative and positive supercoiling domains are transiently generated behind and ahead, respectively, of the moving transcription complex during elongation (Liu and Wang 1987). In the absence of Topo I, the local negative supercoiling domains accumulate behind the moving RNAP to promote template DNA opening. This appears to facilitate R-loop formation during transcription in topA mutants, and the formation of such $\mathrm{R}$ loops is indeed a major problem linked to growth defects observed in top $A$ mutants. Cells stop growth upon inactivation of Topo I unless this occurs in the presence of compensatory mutations. Overexpression of RNase $\mathrm{H}$, which degrades the RNA moiety of an RNA:DNA hybrids, stimulates growth of topA-null mutants, suggesting that R-loop formation is involved in the growth inhibition due to loss of Topo I activity (Drolet et al. 1995). Further studies showed that inactivation of Topo I in fact impairs transcription elongation (Hraiky et al. 2000) and

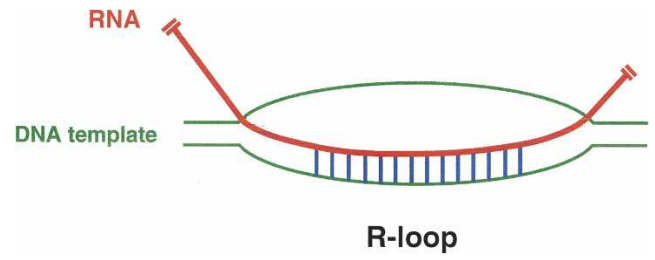

Figure 2. A schematic depiction of an R loop. RNA and DNA strands are indicated. accumulation of full-length RNA (Baaklini et al. 2004), which can again be corrected by RNase $\mathrm{H}$ overexpression. These studies support a model in which excess negative supercoiling in top $A$ mutant cells promotes the formation of cotranscriptional $\mathrm{R}$ loops, which in turn inhibits gene expression and leads to growth defects.

$\mathrm{R}$ loops have also been documented in yeast. Studies by Aguilera and colleagues (Huertas and Aguilera 2003) have demonstrated that cotranscriptional RNA:DNA hybrids exist in Saccharomyces cerevisiae strains containing mutations affecting components of the THO complex. THO is an evolutionarily conserved multisubunit complex, containing in yeast stoichiometric amounts of four polypeptides, Tho2, Hpr1, Mft1, and Thp2 (Chavez et al. 2000). Further studies showed that the THO complex is associated with mRNA export proteins Sub2/UAP56 and Yra1/Aly, forming another conserved complex, the TREX (transcription/export) complex (Strasser et al. 2002; Rehwinkel et al. 2004; Masuda et al. 2005). Although its precise function is not yet clear, the TREX complex has been thought to play critical roles in transcription elongation, mRNA metabolism, and the maintenance of genome integrity. S. cerevisiae mutant strains containing defects of any of the TREX subunits show similar transcription elongation and mRNA export defects, as well as, significantly, transcription-dependent hyperrecombination phenotypes (Prado et al. 1997; Piruat and Aguilera 1998; Chavez et al. 2000; Fan et al. 2001; Jimeno et al. 2002; Strasser et al. 2002; Rondon et al. 2003). For example, a null mutation of hpr1, initially identified as enhancing mitotic intrachromosomal recombination (Aguilera and Klein 1988), strongly stimulates recombination between DNA repeats and increases levels of plasmid and chromosome instability. Significantly, such hyperrecombination was only observed when transcription went through either the DNA repeats or the intervening regions flanked by the repeats, 
indicating that transcription is necessary for the hyperrecombination phenotypes. Hpr1 mutation also results in RNAP II transcription elongation impairment, which is particularly evident for long or GC-rich DNA sequences (Chavez et al. 2001). Similar to the mutants in the mRNA export machinery, such as sub2 or yral, accumulation of poly(A) RNA was detected in the nucleus in the absence of Hprl, demonstrating a defect in mRNA export (Strasser et al. 2002).

How do defects in the TREX complex lead to impaired transcription elongation and hyperrecombination? Huertas and Aguilera (2003) showed that RNA:DNA hybrids are formed cotranscriptionally in the absence of active Hpr1, and that these structures mediate both transcription elongation impairment and transcription-associated recombination (TAR). They first provided evidence that the nascent mRNA is responsible for the transcription elongation defect. Specifically, they found that selfcleavage of the nascent mRNA, induced by insertion of a hammerhead ribozyme, relieved both the impairment in transcribing long GC-rich templates and TAR in the hpr1s cells. Importantly, they further observed that overexpression of RNase $\mathrm{H}$ suppressed both transcription elongation and hyperrecombination phenotypes caused by Hpr1 inactivation, suggesting that RNA:DNA hybrids formed by nascent RNAs are responsible for the two defects. Consistent with this, by quantitation of the amount of RNase H-sensitive RNA, they provided evidence that $\mathrm{R}$ loops indeed accumulated at high levels in $h p r 1 \Delta$ strains but at much lower levels in wild-type cells as well as hpr1s strains overexpressing RNase H (Huertas and Aguilera 2003). Together, those results indicate that an important function of the TREX complex in yeast is to prevent the formation of cotranscriptional $R$ loops.

$\mathrm{R}$ loops have also been detected in mammals. Notably, they appear to occur naturally in mouse B cells undergoing immunoglobulin class switch recombination (CSR) (Yu et al. 2003; Huang et al. 2006). CSR is a regiondirected gene rearrangement event, resulting in alternative utilization of a variety of immunoglobulin heavychain constant domains (Gritzmacher 1989; Kinoshita and Honjo 2000; Yu and Lieber 2003). CSR junctions form at heterogeneous sites and sequences within or flanking the switch (S) regions (Dunnick et al. 1993; Lee et al. 1998), which are the highly repetitive, and GC-rich regions, transcribed by RNAP II, that extend over several kilobases. Notably, although the precise mechanism by which S regions are recognized by the CSR machinery remains unclear (see below), transcription through the noncoding $S$ regions from corresponding upstream promoters is required for recombination to occur (Jung et al. 1993; Zhang et al. 1993; Bottaro et al. 1994; Harriman et al. 1996). To further elucidate the role of S-region transcription in CSR, Alt and colleagues (Shinkura et al. 2003) developed an embryonic stem (ES) cell-based method to assess the effects of $S$ region mutations on CSR. Their studies demonstrated that inversion of the S region caused a dramatic reduction in CSR, indicating an influence of transcriptional orientation of $S$ regions in
CSR. Significantly, in vitro studies showed that transcription through the $S$ region, but only in its physiological orientation, generates stable RNA:DNA hybrids (Reaban et al. 1994; Tian and Alt 2000; Yu et al. 2003). By employing a sodium bisulfite modification assay to determine the base-pairing status of the two DNA strands, Lieber and his colleagues (Yu et al. 2003; Huang et al. 2006) indeed detected a stable and RNase H-sensitive single-stranded region on the nontemplate DNA strand at the $\mathrm{S}$ regions in mouse B cells undergoing CSR. Altogether, these studies not only indicated the existence of transcriptional $\mathrm{R}$ loops at the $\mathrm{S}$ regions of immunoglobulin genes, but also strongly suggested a cause-effect relationship between R-loop formation and CSR. We note though that additional factors and parallel mechanism(s) may also contribute to CSR targeting (e.g., Chaudhuri et al. 2004; Zarrin et al. 2004).

Recent studies have found that $\mathrm{R}$ loops can form more generally in vertebrate cells under certain abnormal conditions. Specifically, R loops were shown to accumulate in chicken DT40 cells after depletion of the SR /serinearginine) protein splicing factor ASF/SF2 (Li and Manley 2005a). ASF/SF2 is a well-studied SR protein that plays important roles in pre-mRNA splicing and other aspects of RNA metabolism (Ge and Manley 1990; Krainer et al. 1990; Lemaire et al. 2002; Huang et al. 2003; Sanford et al. 2004). However, Li and Manley (2005b) discovered an unexpected role of ASF/SF2 in the maintenance of genome stability. A genetic screen, initially designed to identify proteins that complement the function(s) of ASF/SF2 essential for cell viability, led to the surprising finding that the ASF/SF2-depleted cells became hypermutagenic, likely due to DNA rearrangements. This was reflected in the rapid appearance of double-stranded breaks (DSBs) and high molecular weight (HMW) DNA fragments following loss of ASF/SF2. In the process of pursuing the mechanism by which ASF/SF2 influences genome integrity, it was observed that R loops accumulated in ASF/SF2-depleted cells and were indeed responsible for the observed phenotypes related to genome instability. For example, both HMW DNA fragmentation and hypermutation phenotypes were suppressed by stable overexpression of RNase H. Strikingly, a specific RNase H-sensitive DSB was detected, by ligation-mediated PCR, within a region of the $\beta$-actin gene in which $\mathrm{R}$ loops were generated upon ASF/SF2 depletion. These studies support the idea that inactivation of ASF/SF2 can result in accumulation of cotranscriptional R loops, which in turn lead to DNA DSBs and chromosomal rearrangements (Li and Manley 2005a).

\section{Keeping RNA away from DNA: a common problem leads to diverse solutions throughout evolution}

The involvement of ASF/SF2 in the maintenance of genome stability was initially surprising. SR proteins, including ASF/SF2, have been well documented as splicing factors for more than 15 years (Fu 1995; Manley and Tacke 1996; Graveley 2000). However, given the intrinsic capacity of nascent RNA to displace the nontran- 
scribed DNA strand, forming RNA:DNA hybrid behind the elongating RNAP, a logical way to keep nascent RNA away from DNA might be to package the RNA cotranscriptionally with proteins, such as ASF/SF2, that are involved in processes tightly coupled to transcription.

Retrospectively, the role of cotranscriptional processes in preventing transcriptional R-loop formation has been suggested by several studies in E. coli. As mentioned above, R-loop formation was revealed by the formation of RNase H-sensitive hypernegatively supercoiled away plasmids in topA mutant strains. Significantly such R-loop-dependent supercoiling substantially increases when translation is inhibited (Masse and Drolet 1999; Broccoli et al. 2004). Deletion of the ShineDalgarno sequence (ribosome-binding site) and the ATG initiator codon of the tet $A$ gene resulted in increased R-loop-dependent supercoiling upon transcription of pBR322 in topA-null mutants. Similar RNase H-sensitive supercoiling was also observed in the presence of the protein synthesis inhibitor spectinomycin. Since it has long been known that translation is tightly coupled to transcription in bacteria, the role of translation in preventing R-loop formation can be explained by the presence of ribosomes on the nascent transcript shortly after it emerges from the RNAP, thereby preventing the RNA from reannealing with the template DNA strand. Alternatively, movement of ribosomes along the nascent RNA could disrupt RNA:DNA hybrids that extend from transcriptional bubbles (Fig. 3A, top panel; Drolet et al. 2003; Gowrishankar and Harinarayanan 2004).

In some cases-for example, in the absence of translating ribosomes-E. coli cells can employ an alternative cotranscriptional process, Rho-mediated transcription termination, to prevent transcriptional $\mathrm{R}$ looping. Rho is a ring-shaped, homohexameric protein with RNA-dependent ribonucleoside triphosphatase activity (Roberts 1969). Once bound to RNA, Rho, coupling to the process of ATP hydrolysis, translocates along the nascent RNA in a $5^{\prime} \rightarrow 3^{\prime}$ direction to dissociate the transcript from RNAP and the DNA template, resulting in termination (for reviews, see Nudler and Gottesman 2002; Richardson 2002). It has been demonstrated that transcription of a fragment of the $\operatorname{rrn} B$ operon, encoding the untranslated rRNAs, results in R-loop formation both in vitro and in vivo in the absence of Topo I (Masse et al. 1997; Phoenix et al. 1997). By disrupting the rrnB antitermination system, and thereby activating Rho-dependent termination, R-loop-dependent supercoiling was strongly reduced, pointing to a significant role for Rho-mediated termination in preventing transcriptional $\mathrm{R}$ looping in the absence of Topo I (Fig. 3A, bottom panel; Broccoli et al. 2004).

Transcription and translation are, of course, uncoupled in eukaryotes. Nevertheless, newly synthesized transcripts encounter numerous protein factors, beginning immediately upon emerging from RNAP II, and exist as ribonucleoprotein (RNP) complexes (for reviews, see Daneholt 2001; Dreyfuss et al. 2002). For example, the splicing factor U2AF65 is capable of interacting in vitro with newly initiated RNA as soon as the nascent transcript extends 26-28 nucleotides (Ujvari and Luse 2004). Several recent studies, both from yeast and vertebrate cells, have suggested that cotranscriptional packaging of nascent RNAs into RNP complexes, which are frequently multifunctional, provides an efficient way to keep RNA away from DNA, thereby preventing R-loop formation and the ensuing genomic instability during gene expression.

The observation of cotranscriptionally formed RNA: DNA hybrids in hpr1s mutant yeast cells has implied a crucial role for the THO complex in prevent R-loop formation in yeast (Huertas and Aguilera 2003). The THO complex is cotranscriptionally recruited to transcribed genes and travels along their entire length with RNAP II during transcription (Strasser et al. 2002). Notably, a more detailed chromatin immunoprecipitation analysis has indicated that the recruitment of the THO complex to the yeast gene is insensitive to RNase treat-

A

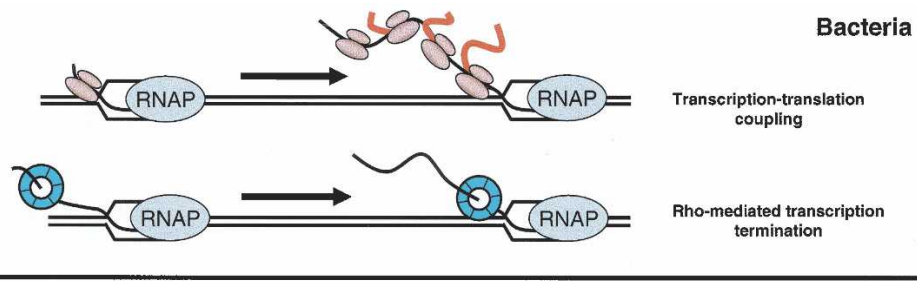

B

Yeast

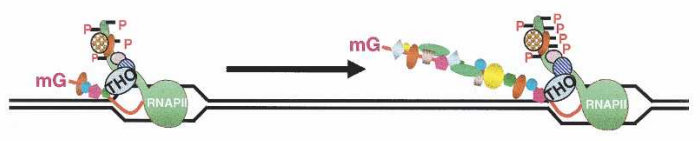

C

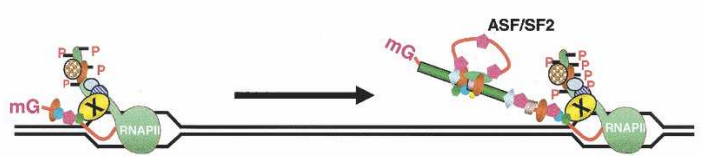

Figure 3. Preventing transcriptional $\mathrm{R}$ loops by diverse mechanisms. (A) In bacteria, two mechanisms, transcription-translation coupling (top panel) and Rho factor-mediated transcription termination (bottom panel), have been suggested to function in preventing transcriptional R-loop formation by preventing or disrupting RNA:DNA hybrids. $(B)$ In yeast, the THO complex (blue oval) functions at the interface between transcription and mRNP assembly and facilitates the cotranscriptional loading of RNA-binding proteins onto nascent transcripts, preventing transcriptional $\mathrm{R}$ looping. $(C)$ In metazoans, SR proteins such as ASF/ SF2 (red pentagon) associate with nascent RNA, either to participate in RNP formation or to recruit the splicing machinery. Bacterial RNAP and RNAP II in yeast and metazoans are indicated. 
ment, suggesting that the THO complex does not associate directly with the nascent RNA (Abruzzi et al. 2004). This raises questions concerning the mechanism by which THO implements its function in keeping the nascent mRNA apart from the DNA during transcription. A possible answer might be that the complex acts at the interface between transcription and mRNP assembly, facilitating the cotranscriptional loading of RNAbinding proteins onto nascent transcripts (Fig. 3B). This view is supported by the observation that the THO complex is required for recruitment of mRNA export factors, such as Sub2 and Yra1, to the nascent RNA (Abruzzi et al. 2004), and both sub2 and yra1 mutants show transcription elongation and TAR phenotypes reminiscent of those observed in the THO complex mutants (Fan et al. 2001; Jimeno et al. 2002). Indeed, overexpression of Sub2 can suppress genome instability phenotypes of hpr1 mutant strains (Fan et al. 2001). On the other hand, although the effects are not as strong and the detailed mechanism still needs to be investigated, it is also notable that hyperrecombination and transcription impairment of a long, GC-rich exogenous lac Z gene are common phenotypes of a collection of mutants functioning in pre-mRNA 3'-end cleavage/polyadenylation, the nuclear exosome, and mRNA export (Luna et al. 2005). Moreover, certain mutations influencing premRNA 3 '-end processing and mRNA decay cause inviability in a tho2 genetic background, indicating the functional interactions between these mRNP processing factors and the THO complex (Luna et al. 2005). These studies also support the view that an interdependence between transcription and mRNP maturation can have a significant impact on genomic stability, and that the THO complex may function as a key integrator in this process.

The same symphony appears to be orchestrated in a different manner in metazoans. In contrast to the tight association of the THO/TREX complex with the transcription machinery in yeast, recruitment of THO/TREX to transcribed genes in mammals is not directly coupled to transcription, and appears to occur in a splicing-dependent manner (Masuda et al. 2005). Antibodies to human THO/TREX components only efficiently immunoprecipitated spliced mRNA but not unspliced premRNA in in vitro reactions, and coupling splicing with RNAP II transcription was unable to facilitate loading of human THO subunits onto the spliced mRNA. While this may reflect the difference in components of the THO/TREX complex between yeast and metazoans (Chavez et al. 2000; Rehwinkel et al. 2004; Masuda et al. 2005), it may also be a consequence of the near ubiquitous presence of introns in metazoan genes. This, together with the tight coupling between transcription and splicing, perhaps makes splicing factors more suitable candidates to participate directly in RNP assembly on nascent transcripts, and thereby to prevent R-loop formation during transcription.

The role of splicing factors in cotranscriptional mRNP assembly has been suggested by a large body of work from Daneholt and colleagues (Daneholt 2001). These investigators have found that, in salivary gland cells in the larvae of the dipteran Chironomus tentans, Hrp45 and Hrp23, two SR protein-like RNA-binding proteins, are cotranscriptionally present along the entire length of Balbani ring nascent transcripts. Because these patterns are distinct from the behavior of spliceosomal snRNP proteins, which tend to localize around the borders of introns and exons, it was proposed that, besides their potential role in splicing, Hrp45 and Hrp23 function as important structural components for pre-mRNP particles (Daneholt 2001). In the current context, it is especially noteworthy that Hrp45 appears to be the homolog of ASF/SF2. Although further investigation is required, the same mechanism might apply in other metazoan organisms, and thereby, as occurs in ASF/SF2-depleted cells, contribute to preventing the formation of cotranscriptional R loops. In any event, these findings support the idea that a splicing-related mechanism might contribute to maintaining the separation between nascent RNA and DNA in metazoans (Fig. 3C).

An intriguing question is how the wrapping of DNA in chromatin affects the propensity to nascent transcripts to form cotranscriptional $\mathrm{R}$ loops. While direct evidence addressing this is limited, some recent studies in yeast have revealed links between chromatin structure, transcription elongation, and genome stability. For example, Winston and colleagues (Nourani et al. 2006) demonstrated that the protein Spt2/Sin1, a DNA-binding protein that functions in regulation of chromatin structure and transcription, is required for repression of intrachromosomal recombination. Spt2/Sin1 encodes a protein with similarities to mammalian HMG1, a structural component of chromatin (Kruger and Herskowitz 1991). In the process of elucidating the precise role of Spt2/Sin1, Nourani et al. (2006) found that the frequency of recombination between inverted repeats was significantly increased in spt2 $2 \Delta$ mutant cells. Interestingly, similar to hpr1s mutants, such hyperrecombination is transcription-dependent. The hyperrecombination phenotype was significantly impaired when the level of transcription toward the inverted repeats was reduced in the spt $2 \Delta$ mutant cells. However, it is unclear whether R loops are induced upon Spt2/Sin 1 inactivation, and it also needs to be determined whether alteration of chromatin structure is directly responsible for genome instability resulting from loss of Spt2/ Sin1.

Additional chromosome-associated proteins may also contribute to R-loop formation. For example, singlestrand DNA (ssDNA)-binding proteins may facilitate RNA:DNA hybrid formation during transcription by stabilizing the single-standed region on the nontemplate strand. Chaudhuri et al. (2004) suggested that replication protein A (RPA), a ubiquitous ssDNA-binding protein, helps activation induced deaminase (AID; see below) deaminate deoxycytidines during immunoglobulin somatic hypermutation (SHM) targets, most likely by stabilizing ssDNA at small, transient transcription bubbles. Following this line of reasoning, it will be of great interest to determine whether RPA, as well as other ssDNA- 
binding protein(s), plays roles in $\mathrm{R}$ loop formation by stabilizing single-stranded regions.

\section{How do transcriptional $\mathrm{R}$ loops induce genome instability?}

Transcriptional R-loop formation in eukaryotes is highly correlated with DNA recombination and/or impairment of genome stability, indicating an inherent impact of $\mathrm{R}$ looping on the integrity of the genome. Although relatively little is known about the molecular mechanism(s) by which transcriptional $\mathrm{R}$ loops influence genome stability, several possible scenarios can be envisioned.

It has long been known that ssDNA is more vulnerable to mutations than dsDNA (e.g., Lindahl and Nyberg 1974). Thus, in one model, extensive R-loop formation may make certain transcribed regions of the genome more susceptible to DNA-damaging agents simply by increasing the frequency of single-stranded regions (Fig. 4A). This idea is consistent with early observations that transcription synergistically increases the mutagenic effects of DNA-damaging agents in bacteria (e.g., Brock 1971; Herman and Dworkin 1971). More recently, Garcia-Rubio et al. (2003) provided evidence that transcription can also synergistically increase 4-nitroquinoline$\mathrm{N}$-oxide or methyl methanesulfonate-induced recombination in yeast. However, it is unclear whether effects such as these are in fact due to R-loop formation. It will be intriguing in the future to determine, for example, if increased mutagenesis is enhanced in top $A$ mutants or decreased by RNase H overexpression.

In a second scenario, (a) protein factor(s) that specifically recognize(s) R-loop structures might be involved in initiating the generation of mutagenic/recombinant DNA lesions. One candidate is AID, which was first identified as a factor specifically expressed in germinal center B cells upon activation (Muramatsu et al. 1999). Later studies have demonstrated that AID is indeed a key player necessary for CSR (Muramatsu et al. 2000; Okazaki et al. 2002; Longerich et al. 2006). Two models, the RNA-editing model and the DNA-deamination model, have been suggested to explain AID action in CSR. Since the high homology of the amino acid sequence between AID and apolipoprotein B mRNA-editing catalytic polypeptide 1 (APOBEC-1), AID was initially proposed to be an RNA-editing enzyme that modifies an mRNA encoding an unknown endonuclease, which then specifically targets the S regions (Muramatsu et al. 1999, 2000). While this model has not been ruled out, later studies have provided more and more evidence supporting a DNA-based function for AID in CSR. That is, AID directly deaminates cytidine to generate uracil on the DNA, and the resulting U:G mismatch is then processed by the base excision repair components, such as uracil DNA glycosylase (UNG) and apurinic/apyrimidinic endonuclease (APE), to generate a DNA nick in the switch region (Fig. 4B; Di Noia and Neuberger 2002; PetersenMahrt et al. 2002; Rada et al. 2002). The nicks are then further converted into DSBs by mismatch repair proteins to initiate CSR (Stavnezer and Schrader 2006). In this view, it is especially noteworthy that AID prefers to deaminate cytidine in ssDNA /Chaudhuri et al. 2003; Dickerson et al. 2003), and transcription enhances AIDmediated cytidine deamination on the nontemplate strand (Ramiro et al. 2003; Sohail et al. 2003). These studies support a model by which the ssDNA regions resulting from transcriptional $\mathrm{R}$ looping form targets for AID, and this confers upon AID the specificity to introduce DNA breaks in the $S$ region, which initiate the process of CSR.

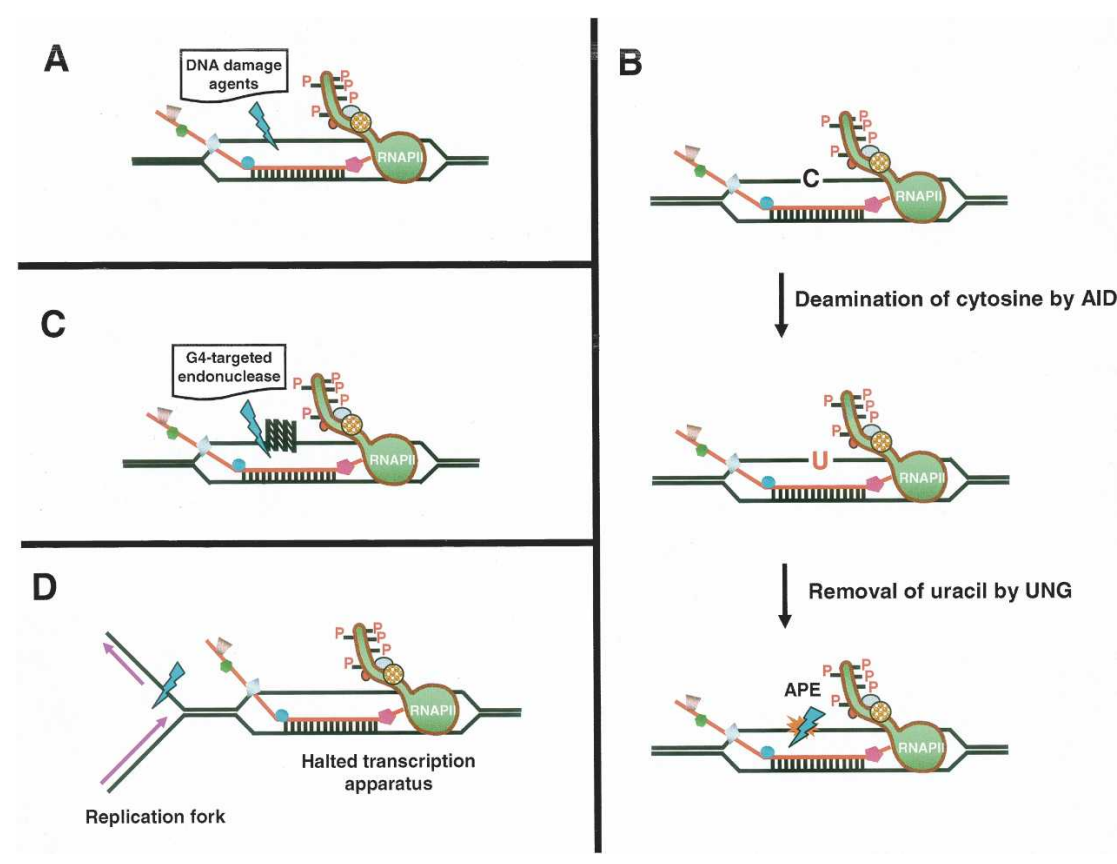

Figure 4. Potential models of transcriptional R-loop-mediated DNA damage. (A) R loops make the genome more susceptible to DNA-damaging agents by increasing the frequency of single-stranded regions. (B) AIDmediated DNA cytosine deamination generates abasic sites on the nontemplated DNA strand within R-loop regions. These lead to DNA lesions, catalyzed, for example, by the base excision repair components UNG and APE, which generate a DNA nick in the Rloop region. $(C) \mathrm{G}$-quartets form on the nontemplate DNA strand, and may both contribute to R-loop formation and generate susceptible sites for specific nucleases. $(D)$ The collision between the DNA replication apparatus and transcription elongation complex leads to the formation of DNA lesions. RNAP II is indicated. 
An interesting question is whether AID or a related enzyme might also be responsible for initiating the DNA DSBs observed following ASF/SF2 depletion. In keeping with its role in CSR and other immunoglobulin gene alterations, AID is specifically expressed in germinal B cells. While DT40 cells are B cell derived and express AID, HMW DNA fragments have also been observed in HeLa cells upon small interfering RNA (siRNA)-mediated depletion of ASF/SF2 (Li and Manley 2005a). One possibility is that other APOBEC family members might function analogously to AID in other cell types. Indeed, a number of additional members of this family, expressed in a variety of tissues, have been described, and show DNA deamination activity but with differential context dependence (Beale et al. 2004)

In addition to ssDNA regions, other structural features of $\mathrm{R}$ loops may also contribute to making these structures targets for certain nucleases. Each R loop contains two duplex-single strand junctions. Several nucleases are capable of recognizing such loop-duplex junctions. For example, XPF-ERCC1 and XPG, originally identified as nucleotide excision repair nucleases, are able to cleave bubble structures at the $5^{\prime}$ and $3^{\prime}$ side of the loop-duplex junction, respectively. Intriguingly, it has been reported that recombinant XPF-ERCC1 and XPG can cleave transcribed S regions (Tian and Alt 2000).

It is also interesting to note that transcriptional $\mathrm{R}$ looping occurs preferentially when the nontemplate strand is G rich (e.g., Phoenix et al. 1997; Huertas and Aguilera 2003; Yu et al. 2003; Li and Manley 2005a). Recent studies have indeed shown that guanine is indispensable for the formation of a stable RNA:DNA hybrids in the immunoglobulin $S$ region during in vitro transcription (Duquette et al. 2004; Mizuta et al. 2005). Substitution of GTP with analogs, such as 7-deaza guanine or ITP, impair the formation of higher-order RNA:DNA complexes, presumably $\mathrm{R}$ loops, in the $\mathrm{S}$ region. This is consistent with the fact that the exceptional stability of the $\mathrm{rG} / \mathrm{dC}$ base pairs (Sugimoto et al. 1995) facilitates R-loop formation by favoring RNA:DNA over DNA:DNA hybrids in regions where the nontemplate strand is G-rich, such as the S regions (Gritzmacher 1989). On the other hand, the singlestranded G-rich regions are also prone to form stable parallel four-stranded DNA structures, referred to as Gquartets or G4 DNAs (for review, see Gilbert and Feigon 1999|. This could help stabilize the single-strand nature of the nontemplate strand and thereby facilitate RNA:DNA hybrid formation during transcription. Formation of G-quartet structures during in vitro transcription of $\mathrm{S}$ regions has in fact been observed by electron microscopy, and they have also been detected in $S$ region-containing plasmids propagated in E. coli (Duquette et al. 2004). Notably, several nucleases have been identified, from yeast and mammalian cells, that cleave in the single-stranded region $5^{\prime}$ of stacked G-quartets with striking specificity (Liu et al. 1993; Liu and Gilbert 1994; Sun et al. 2001). These observations raise the possibility that higher-order DNA structures, such as G-quartets, may both help drive the formation of transcriptional $R$ loops and also generate susceptible sites for specific nucleases, although further work is required to determine whether such structures indeed play significant roles in vivo.

An additional possible mechanism for R-loop-mediated DNA damage involves DNA replication. Specifically, transcription elongation complexes halted or slowed by R-loop structures might block the progress of replication forks and lead to DNA recombination and/or DNA DSBs in the newly replicated DNA (Fig. 4D; Aguilera 2002). Given that cotranscriptional R loops have been known to be responsible for the transcription elongation defects in yeast THO mutants (Huertas and Aguilera 2003), it is plausible that resulting stalled elongation complexes could interfere with the replication machinery. In fact, transcription-dependent replication fork collisions have been shown to induce recombination in bacteria and yeast (French 1992; Krasilnikova et al. 1998; Takeuchi et al. 2003). Recently, using plasmid-borne direct-repeat constructs, Prado and Aguilera (2005) provided evidence that a clash between transcription and replication facilitates TAR. A significant increase in TAR was observed when RNAP II transcription encountered a head-on oncoming replication fork, and this was concomitant with the appearance of a replication fork pause. These results support the idea that TAR can be a consequence of replication fork collapse caused by RNAP II-mediated transcription, at least in yeast. Evidence for this phenomenon in vertebrate systems, however, is currently lacking. In addition, ASF/SF2 depletion does not appear to cause an overall decrease in RNAP II transcription rate (Wang et al. 1996). Thus, the idea that transcriptional R-loop formation interferes with replication fork progression and thereby induces DNA damage and genome instability is an intriguing possibility, but one that requires further work.

\section{Concluding remarks}

The studies described above indicate that transcriptioninduced R-loop formation is an intrinsic threat to genome integrity throughout evolution. Regardless of the diversity with respect to the detailed mechanism(s), different species have employed a variety of cotranscriptional processes to prevent the formation of $\mathrm{R}$ loops. In metazoans, the function of ASF/SF2 in preventing R-loop formation suggests an important role of cotranscriptional RNA processing and/or packaging in the maintenance of genome stability during gene expression. However, further investigation is required to determine the detailed mechanism by which $\mathrm{R}$ loops form during transcription. For example, do they initiate directly from the transcription bubble? Or by reannealing of the nascent RNA with the template DNA strand after RNA displacement? Do any other RNA-processing factors and/or nuclear RNA-binding proteins play similar role(s) in preventing R-loop formation? What is the mechanism by which these factors function to prevent $\mathrm{R}$ looping? Is it simply by packaging the RNA into RNP, or does it involve, in fact, removing the RNA by splicing? Studies to 
pursue these questions will contribute to a comprehensive view of the mechanisms involved in protecting the genome from the damaging effects of nascent transcripts during transcription.

Another critical unresolved issue is how transcriptional R loops impacts genome integrity. Although the issue has not yet been addressed in bacteria, the studies described above indicate that R-loop formation is capable of inducing hyperrecombination and/or hypermutation phenotypes in eukaryotes (see Aguilera 2005a,b). Several scenarios have been proposed above to explain how R loops mediate DNA damage and ultimately genome instability. However, detailed dissection of the mechanism(s) and the factor(s) involved in R-loop formation will be required to test these various possibilities. How is a DNA lesion introduced into the R-loop region? What kinds of DNA damage tolerance pathways are activated by R-loop-induced DNA damage? How do they in turn lead to DNA rearrangements and/or recombination? As an extension of these ideas, it will be of great interest to examine whether transcription-induced R-loop formation contributes to any disease-related genetic alterations. Considering the high correlation between genetic instability and tumorgenesis, investigation of the potential role in cancer of the relevant cotranscriptional processes, such as mRNP formation, that prevent formation of $\mathrm{R}$ loops will be an attractive topic for future research.

\section{References}

Abruzzi, K.C., Lacadie, S., and Rosbash, M. 2004. Biochemical analysis of TREX complex recruitment to intronless and intron-containing yeast genes. EMBO J. 23: 2620-2631.

Aguilera, A. 2002. The connection between transcription and genomic instability. EMBO I. 21: 195-201.

- 2005a. Cotranscriptional mRNP assembly: From the DNA to the nuclear pore. Curr. Opin. Cell Biol. 17: 242-250. . 2005b. mRNA processing and genomic instability. Nat. Struct. Mol. Biol. 12: 737-738.

Aguilera, A. and Klein, H.L. 1988. Genetic control of intrachromosomal recombination in Saccharomyces cerevisiae. I. Isolation and genetic characterization of hyper-recombination mutations. Genetics 119: 779-790.

Baaklini, I., Hraiky, C., Rallu, F., Tse-Dinh, Y.C., and Drolet, M. 2004. RNase HI overproduction is required for efficient fulllength RNA synthesis in the absence of topoisomerase I in Escherichia coli. Mol. Microbiol. 54: 198-211.

Beale, R.C., Petersen-Mahrt, S.K., Watt, I.N., Harris, R.S., Rada, C., and Neuberger, M.S. 2004. Comparison of the differential context-dependence of DNA deamination by APOBEC enzymes: Correlation with mutation spectra in vivo. J. Mol. Biol. 337: 585-596.

Bottaro, A., Lansford, R., Xu, L., Zhang, J., Rothman, P., and Alt, F.W. 1994. S region transcription per se promotes basal IgE class switch recombination but additional factors regulate the efficiency of the process. EMBO J. 13: 665-674.

Broccoli, S., Rallu, F., Sanscartier, P., Cerritelli, S.M., Crouch, R.J., and Drolet, M. 2004. Effects of RNA polymerase modifications on transcription-induced negative supercoiling and associated R-loop formation. Mol. Microbiol. 52: 1769-1779.

Brock, R.D. 1971. Differential mutation of the $\beta$-galactosidase gene of Escherichia coli. Mutat. Res. 11: 181-186.
Chaudhuri, J., Tian, M., Khuong, C., Chua, K., Pinaud, E., and Alt, F.W. 2003. Transcription-targeted DNA deamination by the AID antibody diversification enzyme. Nature 422: 726730.

Chaudhuri, J., Khuong, C., and Alt, F.W. 2004. Replication protein A interacts with AID to promote deamination of somatic hypermutation targets. Nature 430: 992-998.

Chavez, S., Beilharz, T., Rondon, A.G., Erdjument-Bromage, H., Tempst, P., Svejstrup, J.Q., Lithgow, T., and Aguilera, A. 2000. A protein complex containing Tho2, Hpr1, Mft1 and a novel protein, Thp2, connects transcription elongation with mitotic recombination in Saccharomyces cerevisiae. EMBO J. 19: 5824-5834.

Chavez, S., Garcia-Rubio, M., Prado, F., and Aguilera, A. 2001. Hprl is preferentially required for transcription of either long or G + C-rich DNA sequences in Saccharomyces cerevisiae. Mol. Cell. Biol. 21: 7054-7064.

Daneholt, B. 2001. Assembly and transport of a premessenger RNP particle. Proc. Natl. Acad. Sci. 98: 7012-7017.

Di Noia, J. and Neuberger, M.S. 2002. Altering the pathway of immunoglobulin hypermutation by inhibiting uracil-DNA glycosylase. Nature 419: 43-48.

Dickerson, S.K., Market, E., Besmer, E., and Papavasiliou, F.N. 2003. AID mediates hypermutation by deaminating single stranded DNA. J. Exp. Med. 197: 1291-1296.

Dreyfuss, G., Kim, V.N., and Kataoka, N. 2002. MessengerRNA-binding proteins and the messages they carry. Nat. Rev. Mol. Cell Biol. 3: 195-205.

Drlica, K. 1992. Control of bacterial DNA supercoiling. Mol. Microbiol. 6: 425-433.

Drolet, M. 2006. Growth inhibition mediated by excess negative supercoiling: The interplay between transcription elongation, R-loop formation and DNA topology. Mol. Microbiol. 59: 723-730.

Drolet, M., Bi, X., and Liu, L.F. 1994. Hypernegative supercoiling of the DNA template during transcription elongation in vitro. J. Biol. Chem. 269: 2068-2074.

Drolet, M., Phoenix, P., Menzel, R., Masse, E., Liu, L.F., and Crouch, R.J. 1995. Overexpression of RNase H partially complements the growth defect of an Escherichia coli $\Delta$ topA mutant: R-loop formation is a major problem in the absence of DNA topoisomerase I. Proc. Natl. Acad. Sci. 92: 3526-3530.

Drolet, M., Broccoli, S., Rallu, F., Hraiky, C., Fortin, C., Masse, E., and Baaklini, I. 2003. The problem of hypernegative supercoiling and R-loop formation in transcription. Front. Biosci. 8: d210-d221.

Dunnick, W., Hertz, G.Z., Scappino, L., and Gritzmacher, C. 1993. DNA sequences at immunoglobulin switch region recombination sites. Nucleic Acids Res. 21: 365-372.

Duquette, M.L., Handa, P., Vincent, J.A., Taylor, A.F., and Maizels, N. 2004. Intracellular transcription of G-rich DNAs induces formation of G-loops, novel structures containing G4 DNA. Genes \& Dev. 18: 1618-1629.

Fan, H.Y., Merker, R.J., and Klein, H.L. 2001. High-copy-number expression of Sub2p, a member of the RNA helicase superfamily, suppresses hprl-mediated genomic instability. Mol. Cell. Biol. 21: 5459-5470.

French, S. 1992. Consequences of replication fork movement through transcription units in vivo. Science 258: 1362-1365.

$\mathrm{Fu}$, X.D. 1995. The superfamily of arginine/serine-rich splicing factors. RNA 1: 663-680.

Ge, H. and Manley, J.L. 1990. A protein factor, ASF, controls cell-specific alternative splicing of SV40 early pre-mRNA in vitro. Cell 62: 25-34.

Garcia-Rubio, M., Huertas, P., Gonzalez-Barrera, S., and Ag- 
uilera, A. 2003. Recombinogenic effects of DNA-damaging agents are synergistically increased by transcription in Saccharomyces cerevisiae. New insights into transcription-associated recombination. Genetics 165: 457-466.

Gilbert, D.E. and Feigon, J. 1999. Multistranded DNA structures. Curr. Opin. Struct. Biol. 9: 305-314.

Gnatt, A.L., Cramer, P., Fu, J., Bushnell, D.A., and Kornberg, R.D. 2001. Structural basis of transcription: An RNA polymerase II elongation complex at 3.3 A resolution. Science 292: 1876-1882.

Gowrishankar, J. and Harinarayanan, R. 2004. Why is transcription coupled to translation in bacteria? Mol. Microbiol. 54: 598-603.

Graveley, B.R. 2000. Sorting out the complexity of SR protein functions. RNA 6: 1197-1211.

Gritzmacher, C.A. 1989. Molecular aspects of heavy-chain class switching. Crit. Rev. Immunol. 9: 173-200.

Harriman, G.R., Bradley, A., Das, S., Rogers-Fani, P., and Davis, A.C. 1996. IgA class switch in I $\alpha$ exon-deficient mice. Role of germline transcription in class switch recombination. $J$. Clin. Invest. 97: 477-485.

Herman, R.K. and Dworkin, N.B. 1971. Effect of gene induction on the rate of mutagenesis by ICR-191 in Escherichia coli. J. Bacteriol. 106: 543-550.

Hirose, Y. and Manley, J.L. 2000. RNA polymerase II and the integration of nuclear events. Genes \& Dev. 14: 1415-1429.

Hraiky, C., Raymond, M.A., and Drolet, M. 2000. RNase H overproduction corrects a defect at the level of transcription elongation during rRNA synthesis in the absence of DNA topoisomerase I in Escherichia coli. I. Biol. Chem. 275: 11257-11263.

Huang, Y., Gattoni, R., Stevenin, J., and Steitz, J.A. 2003. SR splicing factors serve as adapter proteins for TAP-dependent mRNA export. Mol. Cell 11: 837-843.

Huang, F.T., Yu, K., Hsieh, C.L., and Lieber, M.R. 2006. Downstream boundary of chromosomal R-loops at murine switch regions: Implications for the mechanism of class switch recombination. Proc. Nat1. Acad. Sci. 103: 5030-5035.

Huertas, P. and Aguilera, A. 2003. Cotranscriptionally formed DNA:RNA hybrids mediate transcription elongation impairment and transcription-associated recombination. Mol. Cell 12: $711-721$.

Itoh, T. and Tomizawa, J. 1980. Formation of an RNA primer for initiation of replication of ColE1 DNA by ribonuclease $\mathrm{H}$. Proc. Nat1. Acad. Sci. 77: 2450-2454.

Jimeno, S., Rondon, A.G., Luna, R., and Aguilera, A. 2002. The yeast THO complex and mRNA export factors link RNA metabolism with transcription and genome instability. EMBO J. 21: 3526-3535.

Jung, S., Rajewsky, K., and Radbruch, A. 1993. Shutdown of class switch recombination by deletion of a switch region control element. Science 259: 984-987.

Kadesch, T.R. and Chamberlin, M.J. 1982. Studies of in vitro transcription by calf thymus RNA polymerase II using a novel duplex DNA template. J. Biol. Chem. 257: 5286-5295.

Kettenberger, H., Armache, K.J., and Cramer, P. 2004. Complete RNA polymerase II elongation complex structure and its interactions with NTP and TFIIS. Mol. Cell 16: 955-965.

Kinoshita, K. and Honjo, T. 2000. Unique and unprecedented recombination mechanisms in class switching. Curr. Opin. Immunol. 12: 195-198.

Krainer, A.R., Conway, G.C., and Kozak, D. 1990. The essential pre-mRNA splicing factor SF2 influences 5 ' splice site selection by activating proximal sites. Cell 62: 35-42.

Krasilnikova, M.M., Samadashwily, G.M., Krasilnikov, A.S., and Mirkin, S.M. 1998. Transcription through a simple
DNA repeat blocks replication elongation. EMBO J. 17: 5095-5102.

Kruger, W. and Herskowitz, I. 1991. A negative regulator of HO transcription, SIN1 (SPT2), is a nonspecific DNA-binding protein related to HMG1. Mol. Cell. Biol. 11: 4135-4146.

Lee, C.G., Kondo, S., and Honjo, T. 1998. Frequent but biased class switch recombination in the $\mathrm{S}$ mu flanking regions. Curr. Biol. 8: 227-230.

Lemaire, R., Prasad, J., Kashima, T., Gustafson, J., Manley, J.L., and Lafyatis, R. 2002. Stability of a PKCI-1-related mRNA is controlled by the splicing factor ASF/SF2: A novel function for SR proteins. Genes \& Dev. 16: 594-607.

Li, X. and Manley, J.L. 2005a. Inactivation of the SR protein splicing factor ASF/SF2 results in genomic instability. Cell 122: 365-378.

. 2005b. New talents for an old acquaintance: The SR protein splicing factor ASF/SF2 functions in the maintenance of genome stability. Cell Cycle 4: 1706-1708.

Lindahl, T. and Nyberg, B. 1974. Heat-induced deamination of cytosine residues in deoxyribonucleic acid. Biochemistry 13: 3405-3410.

Liu, Z. and Gilbert, W. 1994. The yeast KEM1 gene encodes a nuclease specific for G4 tetraplex DNA: Implication of in vivo functions for this novel DNA structure. Cell 77: 10831092.

Liu, L.F. and Wang, J.C. 1987. Supercoiling of the DNA template during transcription. Proc. Nat1. Acad. Sci. 84: 7024-7027.

Liu, Z., Frantz, J.D., Gilbert, W., and Tye, B.K. 1993. Identification and characterization of a nuclease activity specific for G4 tetrastranded DNA. Proc. Natl. Acad. Sci. 90: 31573161.

Longerich, S., Basu, U., Alt, F., and Storb, U. 2006. AID in somatic hypermutation and class switch recombination. Curr. Opin. Immunol. 18: 164-174.

Luna, R., Jimeno, S., Marin, M., Huertas, P., Garcia-Rubio, M., and Aguilera, A. 2005. Interdependence between transcription and mRNP processing and export, and its impact on genetic stability. Mol. Cell 18: 711-722.

Maniatis, T. and Reed, R. 2002. An extensive network of coupling among gene expression machines. Nature 416: 499506.

Manley, J.L. and Tacke, R. 1996. SR proteins and splicing control. Genes \& Dev. 10: 1569-1579.

Masse, E. and Drolet, M. 1999. Escherichia coli DNA topoisomerase I inhibits R-loop formation by relaxing transcriptioninduced negative supercoiling. J. Biol. Chem. 274: 1665916664.

Masse, E., Phoenix, P., and Drolet, M. 1997. DNA topoisomerases regulate R-loop formation during transcription of the rrnB operon in Escherichia coli. J. Biol. Chem. 272: 1281612823.

Masuda, S., Das, R., Cheng, H., Hurt, E., Dorman, N., and Reed, R. 2005. Recruitment of the human TREX complex to mRNA during splicing. Genes \& Dev. 19: 1512-1517.

Mizuta, R., Mizuta, M., and Kitamura, D. 2005. Guanine is indispensable for immunoglobulin switch region RNADNA hybrid formation. I. Electron Microsc. (Tokyo) 54: 403-408.

Muramatsu, M., Sankaranand, V.S., Anant, S., Sugai, M., Kinoshita, K., Davidson, N.O., and Honjo, T. 1999. Specific expression of activation-induced cytidine deaminase (AID), a novel member of the RNA-editing deaminase family in germinal center B cells. J. Biol. Chem. 274: 18470-18476.

Muramatsu, M., Kinoshita, K., Fagarasan, S., Yamada, S., Shinkai, Y., and Honjo, T. 2000. Class switch recombination and hypermutation require activation-induced cytidine 
deaminase (AID), a potential RNA editing enzyme. Cell 102: 553-563.

Nourani, A., Robert, F., and Winston, F. 2006. Evidence that Spt2/Sin1, an HMG-like factor, plays roles in transcription elongation, chromatin structure, and genome stability in Saccharomyces cerevisiae. Mol. Cell. Biol. 26: 1496-1509.

Nudler, E. and Gottesman, M.E. 2002. Transcription termination and anti-termination in E. coli genes. Cells 7: 755-768.

Okazaki, I.M., Kinoshita, K., Muramatsu, M., Yoshikawa, K., and Honjo, T. 2002. The AID enzyme induces class switch recombination in fibroblasts. Nature 416: 340-345.

Petersen-Mahrt, S.K., Harris, R.S., and Neuberger, M.S. 2002. AID mutates E. coli suggesting a DNA deamination mechanism for antibody diversification. Nature 418: 99-103.

Phoenix, P., Raymond, M.A., Masse, E., and Drolet, M. 1997. Roles of DNA topoisomerases in the regulation of R-loop formation in vitro. J. Biol. Chem. 272: 1473-1479.

Piruat, J.I. and Aguilera, A. 1998. A novel yeast gene, THO2, is involved in RNA pol II transcription and provides new evidence for transcriptional elongation-associated recombination. EMBO J. 17: 4859-4872.

Prado, F. and Aguilera, A. 2005. Impairment of replication fork progression mediates RNA polII transcription-associated recombination. EMBO J. 24: 1267-1276.

Prado, F., Piruat, J.I., and Aguilera, A. 1997. Recombination between DNA repeats in yeast hprl $\Delta$ cells is linked to transcription elongation. EMBO J. 16: 2826-2835.

Proudfoot, N.J., Furger, A., and Dye, M.J. 2002. Integrating mRNA processing with transcription. Cell 108: 501-512.

Rada, C., Williams, G.T., Nilsen, H., Barnes, D.E., Lindahl, T., and Neuberger, M.S. 2002. Immunoglobulin isotype switching is inhibited and somatic hypermutation perturbed in UNG-deficient mice. Curr. Biol. 12: 1748-1755.

Ramiro, A.R., Stavropoulos, P., Jankovic, M., and Nussenzweig, M.C. 2003. Transcription enhances AID-mediated cytidine deamination by exposing single-stranded DNA on the nontemplate strand. Nat. Immunol. 4: 452-456.

Reaban, M.E., Lebowitz, J., and Griffin, J.A. 1994. Transcription induces the formation of a stable RNA.DNA hybrid in the immunoglobulin $\alpha$ switch region. J. Biol. Chem. 269: 2185021857.

Reed, R. and Cheng, H. 2005. TREX, SR proteins and export of mRNA. Curr. Opin. Cell Biol. 17: 269-273.

Rehwinkel, J., Herold, A., Gari, K., Kocher, T., Rode, M., Ciccarelli, F.L., Wilm, M., and Izaurralde, E. 2004. Genomewide analysis of mRNAs regulated by the THO complex in Drosophila melanogaster. Nat. Struct. Mol. Biol. 11: 558566.

Richardson, J.P. 2002. Rho-dependent termination and ATPases in transcript termination. Biochim. Biophys. Acta 1577: 251-260.

Roberts, J.W. 1969. Termination factor for RNA synthesis. Nature 224: 1168-1174.

Rondon, A.G., Jimeno, S., Garcia-Rubio, M., and Aguilera, A. 2003. Molecular evidence that the eukaryotic THO/TREX complex is required for efficient transcription elongation. $J$. Biol. Chem. 278: 39037-39043.

Sanford, J.R., Gray, N.K., Beckmann, K., and Caceres, J.F. 2004. A novel role for shuttling SR proteins in mRNA translation. Genes \& Dev. 18: 755-768.

Shinkura, R., Tian, M., Smith, M., Chua, K., Fujiwara, Y., and Alt, F.W. 2003. The influence of transcriptional orientation on endogenous switch region function. Nat. Immunol. 4: 435-441.

Sohail, A., Klapacz, J., Samaranayake, M., Ullah, A., and Bhagwat, A.S. 2003. Human activation-induced cytidine deami- nase causes transcription-dependent, strand-biased C to $\mathrm{U}$ deaminations. Nucleic Acids Res. 31: 2990-2994.

Stavnezer, J. and Schrader, C.E. 2006. Mismatch repair converts AID-instigated nicks to double-strand breaks for antibody class-switch recombination. Trends Genet. 22: 23-28.

Strasser, K., Masuda, S., Mason, P., Pfannstiel, J., Oppizzi, M., Rodriguez-Navarro, S., Rondon, A.G., Aguilera, A., Struhl, K., Reed, R., et al. 2002. TREX is a conserved complex coupling transcription with messenger RNA export. Nature 417: 304-308.

Sugimoto, N., Nakano, S., Katoh, M., Matsumura, A., Nakamuta, H., Ohmichi, T., Yoneyama, M., and Sasaki, M. 1995. Thermodynamic parameters to predict stability of RNA/ DNA hybrid duplexes. Biochemistry 34: 11211-11216.

Sun, H., Yabuki, A., and Maizels, N. 2001. A human nuclease specific for G4 DNA. Proc. Natl. Acad. Sci. 98: 12444 12449.

Takeuchi, Y., Horiuchi, T., and Kobayashi, T. 2003. Transcription-dependent recombination and the role of fork collision in yeast rDNA. Genes \& Dev. 17: 1497-1506.

Tian, M. and Alt, F.W. 2000. Transcription-induced cleavage of immunoglobulin switch regions by nucleotide excision repair nucleases in vitro. J. Biol. Chem. 275: 24163-24172.

Ujvari, A. and Luse, D.S. 2004. Newly Initiated RNA encounters a factor involved in splicing immediately upon emerging from within RNA polymerase II. J. Biol. Chem. 279: 4977349779.

Wang, J., Takagaki, Y., and Manley, J.L. 1996. Targeted disruption of an essential vertebrate gene: ASF/SF2 is required for cell viability. Genes \& Dev. 10: 2588-2599.

Westover, K.D., Bushnell, D.A., and Kornberg, R.D. 2004. Structural basis of transcription: Separation of RNA from DNA by RNA polymerase II. Science 303: 1014-1016.

$\mathrm{Yu}, \mathrm{K}$. and Lieber, M.R. 2003. Nucleic acid structures and enzymes in the immunoglobulin class switch recombination mechanism. DNA Repair (Amst.) 2: 1163-1174.

Yu, K., Chedin, F., Hsieh, C.L., Wilson, T.E., and Lieber, M.R. 2003. R-loops at immunoglobulin class switch regions in the chromosomes of stimulated B cells. Nat. Immunol. 4: 442451.

Zarrin, A.A., Alt, F.W., Chaudhuri, J., Stokes, N., Kaushal, D., Du Pasquier, L., and Tian, M. 2004. An evolutionarily conserved target motif for immunoglobulin class-switch recombination. Nat. Immunol. 5: 1275-1281.

Zhang, J., Bottaro, A., Li, S., Stewart, V., and Alt, F.W. 1993. A selective defect in IgG2b switching as a result of targeted mutation of the I $\gamma 2 \mathrm{~b}$ promoter and exon. EMBO J. 12: 3529-3537.

Zorio, D.A. and Bentley, D.L. 2004. The link between mRNA processing and transcription: Communication works both ways. Exp. Cell Res. 296: 91-97. 


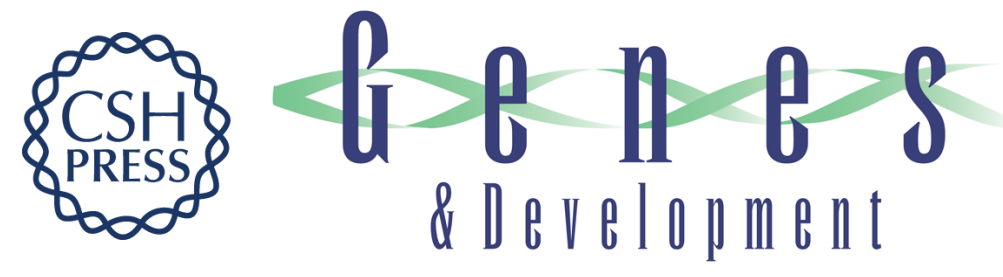

\section{Cotranscriptional processes and their influence on genome stability}

Xialu Li and James L. Manley

Genes Dev. 2006, 20:

Access the most recent version at doi:10.1101/gad.1438306

References This article cites 103 articles, 45 of which can be accessed free at: http://genesdev.cshlp.org/content/20/14/1838.full.html\#ref-list-1

License

Email Alerting Receive free email alerts when new articles cite this article - sign up in the box at the top Service right corner of the article or click here.

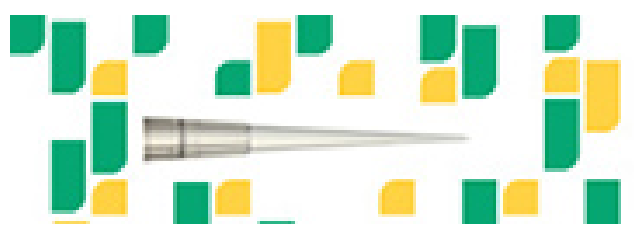

Focused on your science. 